$\underset{\text { clinical }}{\text { nephron }}$

Practice
Nephron 2015;130:13-20

DOI: $10.1159 / 000381480$
Received: January 12, 2015

Accepted after revision: March 6, 2015

Published online: April 21, 2015

\title{
A Multidrug, Antiproteinuric Approach to Alport Syndrome: A Ten-Year Cohort Study
}

\author{
Erica Daina ${ }^{a}$ Paolo Cravedia, d Mirella Alpa ${ }^{b}$ Dario Roccatello ${ }^{b}$ Sara Gamba ${ }^{a}$ \\ Annalisa Perna ${ }^{a}$ Flavio Gaspari ${ }^{a}$ Giuseppe Remuzzi ${ }^{a, c}$ Piero Ruggenenti ${ }^{a, c}$ \\ aIRCCS - Istituto di Ricerche Farmacologiche 'Mario Negri', Clinical Research Centre for Rare Diseases 'Aldo \& Cele Daccò', \\ Bergamo, ${ }^{b}$ CMID, Center of Research on Immunopathology and Rare Diseases, Ospedale S. Giovanni Bosco and University \\ of Torino, Torino, and 'Unit of Nephrology, Azienda Ospedaliera Papa Giovanni XXIII, Bergamo, Italy; ${ }^{d}$ Icahn School of \\ Medicine at Mount Sinai, New York, N.Y., USA
}

\section{Key Words}

ACE inhibitor - Alport syndrome - Angiotensin receptor blocker $\cdot$ Proteinuria $\cdot$ Rare diseases

\begin{abstract}
Background/Aims: Combined ACE inhibitor, angiotensinreceptor-blocker, non-dihydropyridine calcium-channelblocker, and statin therapy (Remission Clinic) reduced proteinuria and halted progression in non-diabetic nephropathies, but their efficacy in Alport syndrome (AS) nephropathy is unknown. Methods: From February 2004 to September 2007, we included nine albuminuric AS adults with creatinine clearance $>20 \mathrm{ml} / \mathrm{min} / 1.73 \mathrm{~m}^{2}$ in a single-center, openlabel, prospective, off-on-off academic study. After the 1-month wash-out from RAS inhibition (Run-in), patients entered the 4-month, add-on, treatment period with benazepril (10-20 mg/day), valsartan (80-160 mg/day), diltiazem (60-120 mg/day), and fluvastatin (40-80 mg/day) followed by the 1-month wash-out (Recovery). The primary outcome was albuminuria at month 4 . After recovery, patients were kept on the Remission Clinic protocol and followed until July 2014 (Extension). Results: The median (IQR) albuminuria progressively declined from $657.7(292.7-1,089.6) \mu \mathrm{g} / \mathrm{min}$ at baseline to $71.4(21.7-504.9) \mu \mathrm{g} / \mathrm{min}$ at treatment end $(p=$ 0.009 ) and raised to $404.3(167.9-446.8) \mu \mathrm{g} / \mathrm{min}$ after recovery. Albumin and IgG fractional clearances significantly ( $p \leq$
\end{abstract}

() 2015 S. Karger AG, Basel

$1660-8151 / 15 / 1301-0013 \$ 39.50 / 0$
0.005) decreased from $66.9(53.6-80.8)$ to $9.4(4.6-26.0)$ and from $5.1(3.0-8.4)$ to $1.1(0.6-3.2)$, and then recovered toward baseline. Blood pressure and lipids significantly decreased on treatment, without changes in inulin-measured GFR or para-aminohippuric-measured RPF. After recovery, one patient refused to enter the extension, one with severe renal insufficiency at baseline reached ESRD, and seven retained normal serum creatinine until the end of the study. At the final visit, three were microalbuminuric and one was normoalbuminuric. Treatment was well tolerated. Conclusion: The Remission Clinic approach safely ameliorated albuminuria, blood pressure, lipids, and glomerular selectivity in AS patients and halted long-term progression in those without renal insufficiency to start with.

๑) 2015 S. Karger AG, Basel

\section{Introduction}

Alport syndrome (AS) is a hereditary type IV collagen disease, which in approximately $50 \%$ of untreated male patients progresses to ESRD by the age of 20 years [1]. There is no specific treatment for AS nephropathy. In animals with autosomal-recessive AS such as COL4A3

E.D. and P.C. contributed equally to the manuscript.

\section{KARGER 125}

E-Mail karger@karger.com www.karger.com/nef
Dr. Giuseppe Remuzzi

IRCCS - Mario Negri Institute for Pharmacological Research Centro Anna Maria Astori, Science \& Technology Park Kilometro Rosso Via Stezzano, 87, IT-24126 Bergamo (Italy)

E-Mail giuseppe.remuzzi@marionegri.it 
knockout mice, preemptive Angiotensin-ConvertingEnzyme (ACE) inhibition with ramipril therapy started at four weeks of age prevented renal fibrosis, postponed uremia, and doubled the lifespan [2]; whereas later intervention started after the onset of proteinuria failed to appreciably affect disease progression and kidney and mice survival [2]. Consistently, data from the European Alport Registry study [3] showed that early ACE inhibitor therapy started at the stage of isolated hematuria or microalbuminuria normalized urinary albumin and slowed down to a very significant extent progression to ESRD in patients with the disease. Conversely, when ACE inhibitor therapy was initiated after the onset of proteinuria or renal insufficiency, proteinuria declined only partially and transiently, and it marginally affected renal outcomes of patients who progressed to ESRD by the age of 25-40 years. Their untreated relatives required dialysis therapy at a median age of 22 years [3]. Thus, both mouse and human data converge to indicate that early treatment allows preventing the onset and progression of chronic kidney disease (CKD); whereas more effective intervention strategies are needed to halt progression in AS patients with established proteinuria.

Evidence is available that an integrated treatment with different medications that lower proteinuria by multiple and potentially synergistic mechanisms might help in achieving this target. Animal [4] and human [5] studies have consistently found that ACE inhibitors and Angiotensin Receptor Blockers (ARBs) share similar antiproteinuric and renoprotective effects. The antiproteinuric effect can be increased by $50-60 \%$ if the two drugs are used in combination $[6,7]$, and combination therapy may stabilize and even improve kidney function in patients with non-diabetic CKD and severe proteinuria who are otherwise predicted to progress to ESRD in a few years while on ACE inhibitor monotherapy $[8,9]$. Non-dihydropyridine calcium channel blockers (ndCCBs) and hydroxymethyl-glutaryl CoA (HMGCoA) inhibitors (statins) have also been reported to reduce proteinuria regardless of their effect on BP [10] or serum lipids [11], respectively, and some data suggested that ndCCBs could also slow renal disease progression over time [10]. A multimodal, individually tailored, intervention strategy, including HMGCoA inhibitors, added on dual RAS blockade to target urinary proteins blunted glomerulosclerosis and prevented progression to ESRD in rats with severe proteinuric CKD [12]. A similar strategy - the Remission Clinic program - was implemented to target urinary proteins in humans by ACE inhibitors and ARBs up-titrated to maximum tolerated doses, plus a statin and an ndCCB
[8]. This individually tailored approach, in addition to achieving remission and even regression of proteinuria, almost fully prevented kidney failure in 56 patients with residual nephrotic-range proteinuria despite ACE inhibitor therapy [8]. Thus, in this study, we sought to investigate the antiproteinuric and long-term renoprotective effect of the Remission Clinic approach in AS patients at increased risk of renal disease progression, and verified whether and to what extent the treatment regimen was tolerated in this context.

\section{Patients and Methods}

A single-center, open-label, prospective, and off-on-off academic study with a long-term extended follow-up was conducted. The study was approved by the Ethics Committee of the Clinical Research Center and was conducted in accordance with the EU Clinical Trial Directive (2001/20/EU), Good Clinical Practice, and the Declaration of Helsinki guidelines. Data quality was overseen by the study monitors of the Clinical Research Center for Rare Diseases 'Aldo \& Cele Daccò' of the IRCCS - Istituto di Ricerche Farmacologiche 'Mario Negri', Bergamo, Italy. The trial is registered at ClinicalTrials.gov with the Identifier: NCT00309257.

\section{Participants}

We included $>18$-year-old consenting subjects with AS, urinary albumin excretion (UAE) rate persistently $>200 \mu \mathrm{g} / \mathrm{min}$ in at least two out of three overnight consecutive urine collections, and a creatinine clearance $>20 \mathrm{ml} / \mathrm{min} / 1.73 \mathrm{~m}^{2}$ with less than $30 \%$ variations in the three months prior to study entry. Diagnosis of AS was based on typical pedigree, clinical features, and biopsy findings in all cases, and it was further confirmed by genetic analyses in five. Subjects on treatment with immunosuppressive drugs, vascular disease of the kidney, incomplete bladder emptying, serum $\mathrm{K}^{+}>5.5 \mathrm{mEq} / \mathrm{l}$ despite optimized acid-base balance, and any clinically relevant condition that might affect completion of the study were excluded as well as breastfeeding or pregnant women, or fertile women with ineffective contraception and subjects unable to provide an informed consent. Patients who in the investigators' judgment could not withdraw any ongoing treatment with ACE inhibitors, ARBs, or statins were not considered.

\section{Baseline Evaluation and Follow-Up}

After a 4-week wash-out period from ACE inhibitors, ARBs, and/or HMGCoA reductase inhibitors and one-week wash-out from ndCCBs (fig. 1), potentially eligible patients had a baseline evaluation of office BP (the mean of 3 values taken 2 min apart at the dominant arm), body weight, routine laboratory tests, overnight UAE (mean of three measurements in consecutive overnight urine specimens collected during the three days before the day visit), and albumin/creatinine $(\mathrm{A} / \mathrm{C})$ ratio measurement in spot morning urine. Those fulfilling the selection criteria had a baseline measurement of their glomerular filtration rate (GFR) and renal plasma flow (RPF) by inulin and para-aminohippuric acid renal clearance techniques [6], respectively. Albumin and IgG fractional clearances were also calculated by standard formulas. Then, pa- 


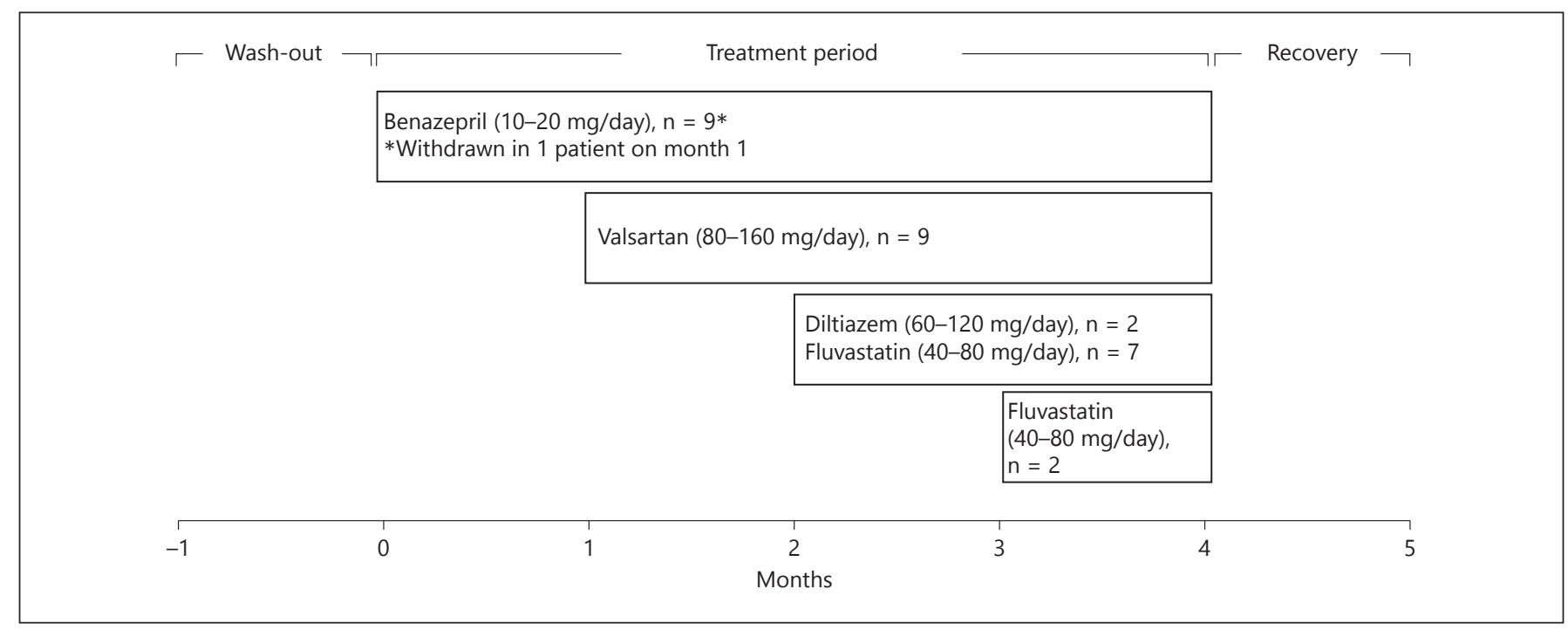

Fig. 1. Study flowchart.

tients entered a study period organized in four consecutive onemonth add-on treatments (month 1: ACE-inhibitor; month 2: ARB; month 3: ndCCB; month 4: HMGco inhibitor) followed by one-month treatment withdrawal (recovery period). Details about treatment doses and stopping rules are provided in the Supplementary material section. At the end of each treatment period and of the recovery period, the measurements of clinical and laboratory parameters considered at baseline were repeated. GFR, RPF, albumin, and IgG fractional clearances were evaluated at the end of the four-month treatment period and of the recovery period (fig. 1).

Detailed descriptions of clearance studies, measurements of serum and urinary protein for fractional clearances, and safety evaluations are available in the online supplementary material section (for all online suppl. material, see www.karger.com/doi/10.1159/ 000381480).

\section{Extension}

After the recovery period, patients were actively followed up until July 2014 or until progression to an endpoint (ESRD or last available visit for those prematurely withdrawing from the study) by 3-6 monthly evaluations of blood pressure, routine laboratory tests, including safety parameters, serum lipids, urinary albumin excretion, and spot urine $\mathrm{A} / \mathrm{C}$ ratio (table 1 ). During this longterm observation period, all of them were maintained on the same treatment regimen that during the treatment period was associated with the largest antiproteinuric effect (Month 4, fig. 1).

\section{Sample Size Estimation}

On the basis of previous evidence [13], in participants with non-nephrotic proteinuria (defined as $24 \mathrm{~h}$ proteinuria $<3.5 \mathrm{~g}$ ), we expected a baseline UAE of approximately $1,000 \mu \mathrm{g} / \mathrm{min}$ and a $70 \%$ reduction to approximately $300 \mu \mathrm{g} / \mathrm{min}$ at 4 months with a standard deviation of the differences equal to $600 \mu \mathrm{g} / \mathrm{min}$. To give the study an $80 \%$ power to detect as statistically significant (alpha $=$ 0.05 , paired t test, two-tailed test) the expected reduction, 8 pa- tients with macroalbuminuria had to be included. Patients with microalbuminuria identified during the recruitment period could also be included as extra-patients.

\section{Data Analysis}

The primary efficacy variable was the change in UAE at the end of the 4-month treatment period versus baseline. Secondary efficacy variables included changes in UAE, spot morning urinary $\mathrm{A} / \mathrm{C}$, systolic/diastolic BP, lipid profile, albumin, and IgG fractional clearances and regression from macro- to micro- or normo- albuminuria at the end of each 1-month treatment period and of the recovery period as compared with baseline. Safety parameters included GFR, RPF, serum creatinine and potassium levels, hemoglobin concentration, and hematocrit.

Data are presented as $\mathrm{n}(\%)$, mean \pm standard deviation (SD), or median and interquartile range (IQR). Wilcoxon signed-rank test or paired t-test or McNemar test was used for a within-group comparison, as appropriate. Longitudinal data analyses of repeated measures over time were performed using a linear mixed-effects model. The UAE and renal hemodynamic and sieving function parameters were log-transformed. All $\mathrm{p}$ values were two-sided. Statistical analyses were performed at the Laboratory of Biostatistics of the Clinical Research Center by using SAS (version 9.1) and STATA (version 12).

\section{Results}

Nine patients (three men) aged 25-69 (median 34) years entered the study from February 24, 2004 to September 9, 2007. At baseline, three of them had heavy albuminuria that in two cases was associated with increased serum creatinine levels. Measured GFR was below normal range $\left(80-120 \mathrm{ml} / \mathrm{min} / 1.73 \mathrm{~m}^{2}\right)$ in five 
Table 1. Patients' clinical and laboratory parameters at baseline (Month 0 ), during the treatment (Month 1 to 4 ) and recovery (Month 5) periods, and at the last available follow-up

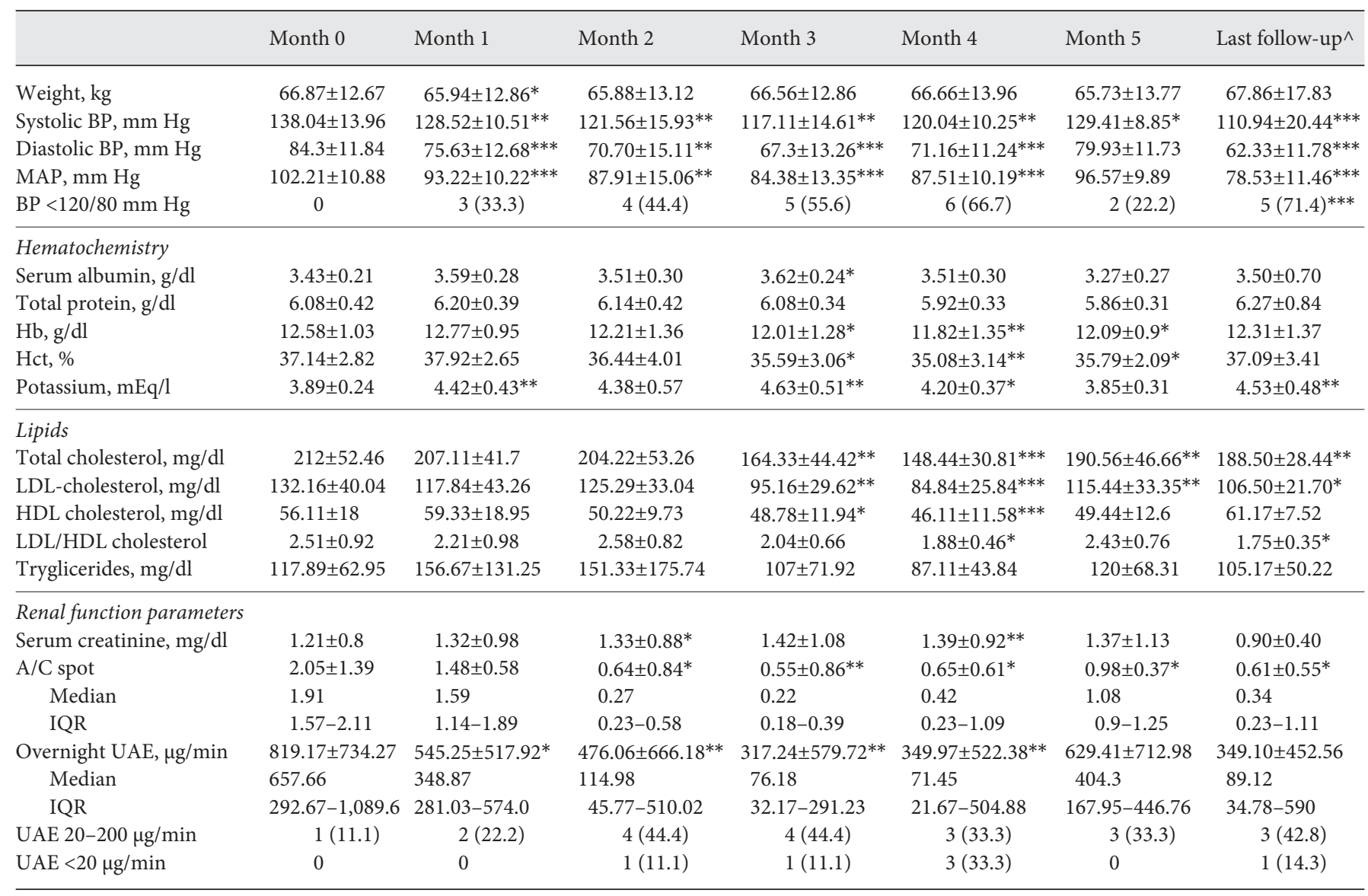

Data are mean $\pm \mathrm{SD}$, median (IQR) or number $(\%) .{ }^{*} \mathrm{p}<0.05,{ }^{* *} \mathrm{p} \leq 0.01,{ }^{* * *} \mathrm{p} \leq 0.001$ vs. month $0 . \wedge$ Include data of the 7 patients on active follow-up who did not reach end stage renal disease.

participants. Three patients were on BP-lowering therapy with carvedilol, clonidine alone, or combined with furosemide, respectively. No patient was on ACE inhibitor or ARB therapy.

\section{Treatment}

All participants received the planned doses of benazepril and valsartan (fig. 1), with the exception of one patient who withdrew the ACE inhibitor at month 1 because of cough. At month 3, five participants did not receive diltiazem, because their BP was already in target $(<120 / 80 \mathrm{~mm} \mathrm{Hg})$ and two because it was contraindicated. As per protocol, these patients received fluvastatin from month 3 and the remaining two participants received it from month 4 . Thus, at month 4 , all patients were on valsartan and fluvastatin therapy; eight were also on benazepril; and two were on diltiazem therapy. Dur- ing the recovery period, all treatments were withdrawn in all participants.

Carvedilol and clonidine were withdrawn during the treatment period. During the recovery period, these treatments were resumed and two additional patients were treated with doxazosin since their BP tended to exceed the recommended safety target $(130 / 90 \mathrm{~mm} \mathrm{Hg})$.

\section{Overnight Albuminuria and Spot Morning A/C Ratio}

During the 4-month treatment period, overnight UAE progressively declined from a median (IQR) of 657.7 $(292.7-1,089.6) \mu \mathrm{g} / \mathrm{min}$ at baseline to $71.4(21.7-504.9)$ $\mu \mathrm{g} / \mathrm{min}$ at the end of the treatment period $(\mathrm{p}=0.009$, table 1). The UAE significantly declined over 1-month benazepril therapy; further progressively decreased though months 2 and 3 when treatment with valsartan $(\mathrm{n}=9)$, diltiazem $(\mathrm{n}=1)$, and fluvastatin $(\mathrm{n}=8)$ was 
Table 2. Renal hemodynamic and sieving function parameters at baseline and at the end of the treatment and recovery periods

\begin{tabular}{lccc}
\hline & Month 0 & Month 4 & Month 5 \\
\hline $\mathrm{GFR}, \mathrm{ml} / \mathrm{min} / 1.73 \mathrm{~m}^{2}$ & $63.27(50.30-95.36)$ & $72.37(37.39-91.71)$ & $85.81(37.73-111.45)$ \\
$\mathrm{RPF}, \mathrm{ml} / \mathrm{min} / 1.73 \mathrm{~m}^{2}$ & $381.50(273.81-462.07)$ & $499.35(181.60-762.78)$ & $452.10(245.97-546.60)$ \\
$\mathrm{eRBF}, \mathrm{ml} / \mathrm{min} / 1.73 \mathrm{~m}^{2}$ & $640.10(402.66-740.50)$ & $784.49(279.88-1,181.53)$ & $684.51(397.30-845.05)$ \\
$\mathrm{FF}(\%)$ & $17.75(11.81-21.50)$ & $14.00(12.14-17.33)$ & $18.29(13.34-19.86)$ \\
$\mathrm{RVR}$ & $0.24(0.22-0.35)$ & $0.18(0.12-0.58)$ & $0.21(0.18-0.57)$ \\
ERVR $^{\circ \circ}$ & $0.15(0.13-0.23)$ & $0.11(0.08-0.37)$ & $0.14(0.12-0.35)$ \\
Alb Fract. Clear. $\left(\times 10^{-5}\right)$ & $66.88(53.63-80.84)$ & $9.38(4.59-25.96)^{*}$ & $47.73(35.16-59.65)$ \\
IgG Fract. Clear $\left(\times 10^{-5}\right)$ & $5.10(3.03-8.44)$ & $1.07(0.55-3.17)^{*}$ & $3.08(2.28-5.03)$
\end{tabular}

Data are mean \pm SD or median (IQR). ${ }^{*} \mathrm{p}<0.01$ vs. month 0 .

${ }^{\circ} \mathrm{eRBF}=\mathrm{RPF} /(1-$ hematocrit $),{ }^{\circ} \mathrm{eRVR}=\mathrm{MAP} / \mathrm{eRBF}$.

added on, respectively; and finally stabilized during month 4 while all participants were on fluvastatin (linear mixed-effects model from baseline to month $4: \mathrm{p}=0.001$ ). Then, the UAE approximated baseline values at the end of the recovery period after all study drugs had been withdrawn for 1 month (table 1; fig. 2, upper panel). At the end of the four-month treatment period, out of the eight participants with macroalbuminuria at inclusion, three had regressed to microalbuminuria and three had regressed to normoalbuminuria (McNemar's test, $\mathrm{p}=0.025$ vs. baseline). At the end of the recovery period, no participant was normoalbuminuric any longer, three were microalbuminuric, and six were macroalbuminuric (table 1). The participant with microalbuminuria at inclusion was persistently microalbuminuric throughout the whole observation period. Albumin/creatinine ratio in spot urine samples followed the same trend of UAE (table 1 and fig. 2, lower panel).

\section{Clearance Studies}

Measured GFR and RPF (or estimated renal blood flow), calculated filtration fraction (FF), and measured or estimated renal vascular resistances (RVR) did not significantly change from baseline to the end of the treatment and recovery periods, whereas albumin and IgG fractional clearances significantly declined at the end of the treatment period $(\mathrm{p}=0.004$ and $\mathrm{p}=0.005$, respectively) and then recovered to baseline values after completion of the recovery period (table 2 and fig. 3 ).

\section{Other Efficacy Parameters}

Systolic, diastolic, and mean arterial BP significantly and progressively declined throughout the 4-month treatment period and then recovered toward baseline values

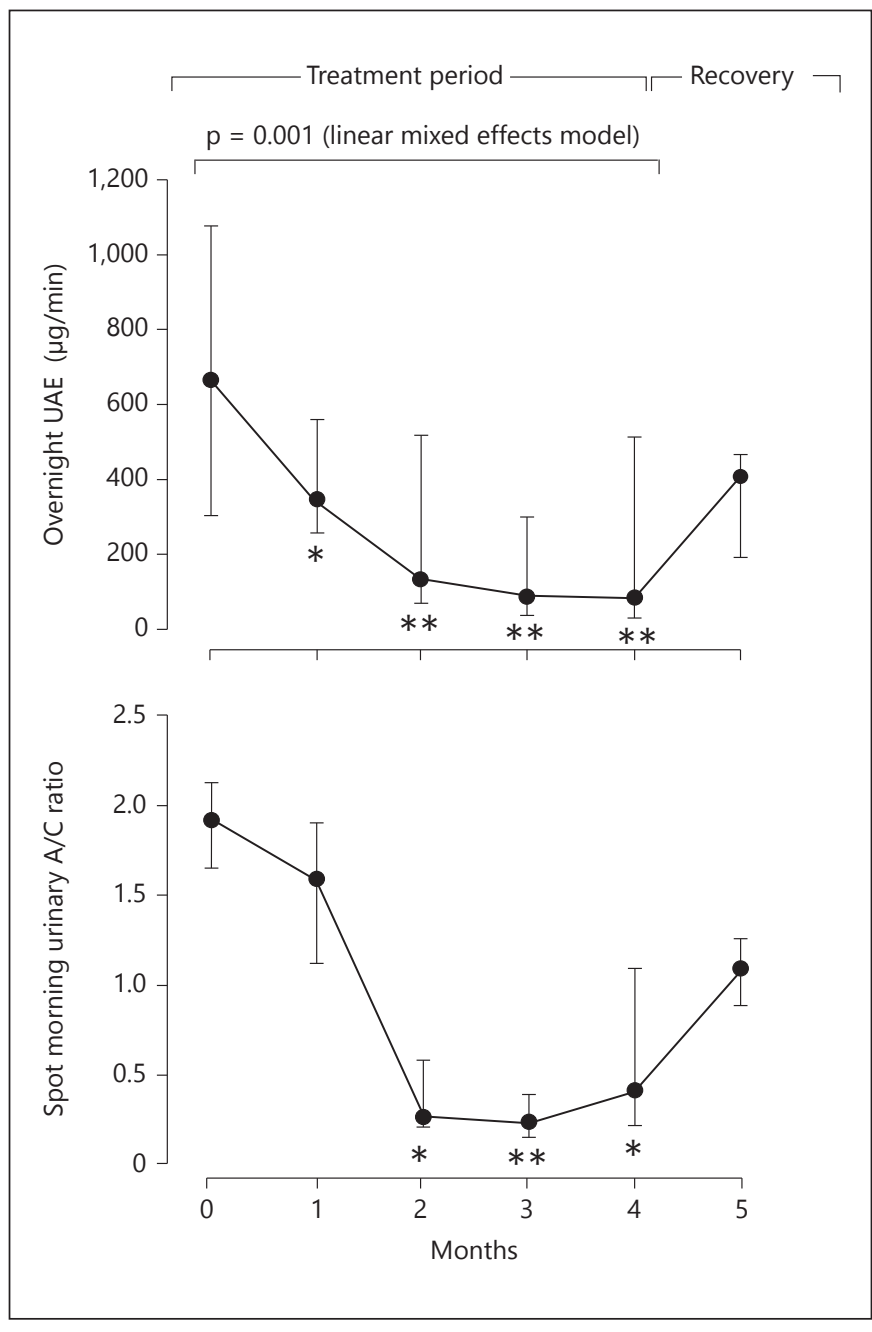

Fig. 2. Changes in overnight UAE (upper panel) and spot morning urinary $\mathrm{A} / \mathrm{C}$ ratio during the treatment and recovery phases as compared to baseline (month 0 ). Data are Median (IQR). ${ }^{*} \mathrm{p}<0.05$ vs. month $0 ;{ }^{* *} \mathrm{p}<0.01$ vs. month 0 and 1 . 

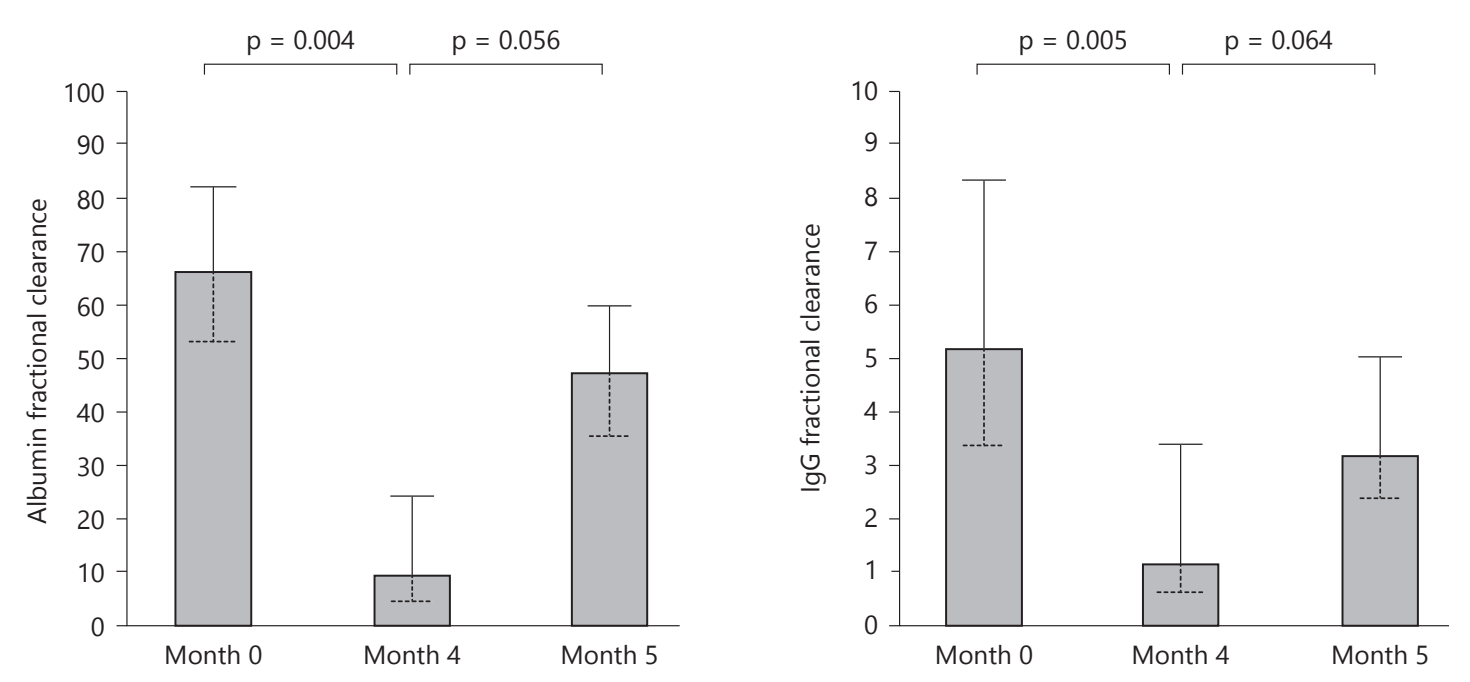

Fig. 3. Albumin (left panel) and IgG (right panel) fractional clearances at baseline and at the end of the 4-month treatment period and of the recovery period. Data are median (IQR).

after treatment withdrawal (table 1). At the end of the treatment period, 6 patients had their BP reduced to $<120 / 80 \mathrm{~mm} \mathrm{Hg}$ compared with none at baseline. Total and LDL cholesterol levels showed a similar trend to progressively decline during the treatment period and partially recovered after treatment withdrawal. HDL cholesterol followed a similar trend; changes, however, were smaller than for LDL cholesterol. Thus, LDL/HDL cholesterol progressively declined during the treatment period and then recovered to baseline values at the study end. No appreciable changes were observed in serum triglycerides, total proteins, and serum albumin levels (table 1).

\section{Sensitivity Analyses}

All considered outcome data did not change appreciably when statistical analyses were performed without considering the patient with microalbuminuria at inclusion (data not shown).

\section{Extension}

After the completion of the recovery period, one patient declined his consensus to continued treatment according to the Remission Clinic program and was lost to follow-up. One of the eight patients maintained on active follow-up progressed to ESRD at 57 months after inclusion. His baseline creatinine and urine spot $\mathrm{A} / \mathrm{C}$ ratio were $2.41 \mathrm{mg} / \mathrm{dl}$ and 1.67, respectively. At 105 (IQR: 92123) months of follow-up, the other seven patients still had normal kidney function. At final visit, their median
UAE and spot urine $\mathrm{A} / \mathrm{C}$ ratio were similar to those observed at the end of the treatment period (month 4, table 1 and fig. 2). Three of these patients had regressed to microalbuminuria and one had regressed to normoalbuminuria (table 1).

\section{Safety}

No patients had acute renal function deterioration, hyperkalemia (serum potassium $>5.5 \mathrm{mEq} / \mathrm{l}$ ), or any other treatment-related serious adverse event throughout the whole observation period. One patient stopped benazepril therapy at month 1 because of cough, while no patient withdrew fluvastatin because of muscle/liver toxicity. Hemoglobin concentration and hematocrit levels slightly but significantly decreased at months 3 and 4 compared to baseline and only partially recovered after treatment withdrawal. Serum potassium increased during the treatment period and then recovered to baseline values at the end of the study.

\section{Discussion}

In this prospective, open-label, single arm, and off-onoff cohort study, we found that a multidrug treatment titrated to urinary albumin - the Remission Clinic protocol - safely and remarkably reduced both overnight albuminuria, the primary efficacy variable of the study, and $\mathrm{A} / \mathrm{C}$ ratio in spot morning urine in 9 albuminuric patients 
with AS. We observed a progressively incremental antiproteinuric effect during the four-month treatment period that weaned after treatment withdrawal. Changes in albuminuria and $\mathrm{A} / \mathrm{C}$ ratios considered as continuous variables were paralleled by a progressive regression from macroalbuminuria to micro- or even normo albuminuria in six participants at the end of the treatment period. Of note, the treatment effect was consistent in all study participants, including those with heavy albuminuria and more severe renal insufficiency and the one single patient who was microalbuminuric at inclusion. Findings that directly measured RPF and GFR did not change appreciably, whereas albumin and IgG fractional clearances remarkably decreased during the treatment period, provided the evidence that the antiproteinuric effect of the Remission Clinic regimen could not be explained by impaired kidney perfusion and filtration, but rather reflected a clinically relevant amelioration of the glomerular sieving dysfunction possibly mediated by decreased intracapillary pressure [14] and/or improved glomerular barrier size selectivity [15]. Independent of mechanism(s), these changes are expected to blunt the ultrafiltration of plasma macromolecules, with amelioration of protein traffic, prevention of protein nephrotoxicity, and protection from progressive kidney damage and function loss [16].

Consistently, over the ten-year study duration, seven out of the eight patients who accepted to enter the extension, long-term treatment period according to the Remission Clinic protocol retained normal renal function, with three patients regressing to microalbuminuria and one to normoalbuminuria. The remaining patient who already had severe renal insufficiency and overt proteinuria at study inclusion eventually progressed to ESRD. Thus, at variance with previous evidence that AS patients with macroalbuminuria progress to ESRD by the age of 40 while on ACE inhibitor monotherapy [3], our present findings confirm that, even at the stage of overt nephropathy, an antiproteinuric approach based on maximized RAS inhibition with ACE inhibitors and ARB combination therapy [8] may halt disease progression - at least in those without severe renal insufficiency to start with and may even achieve remission of proteinuria in a substantial proportion of cases.

Treatment was safe and well tolerated. Only one patient stopped ACE inhibitor therapy because of cough during the treatment period. Serum potassium levels never exceeded $5.5 \mathrm{mEq} / \mathrm{l}$. Thus, treatment is cautiously and gradually up-titrated in parallel with close BP and serum potassium monitoring, the Remission Clinic approach

Antiproteinuric Therapy in Alport

Syndrome can be safely applied to patients with the AS, including those with more advanced renal disease. Importantly, patients continued RAS inhibition at the maximal tolerated doses for more than 6 years with no major adverse effect.

\section{Limitations and Strengths}

This was a small study with no control group. The rarity of the clinical condition prevented identifying enough patients to design a randomized parallel group study comparing the Remission Clinic regimen with ACE inhibitor monotherapy, especially in the context of a singlecenter and resource-limited, fully academic study. We attempted to overcome this limitation by designing a longitudinal study organized in a step-by-step treatment period preceded and followed by run-in and recovery offtreatment periods. With this already described approach [6], each included patient served as his/her-own control. Even more important, finding that after inclusion into the extension phase of the study both albuminuria and spot urine $\mathrm{A} / \mathrm{C}$ ratio again decreased to levels observed at completion of the treatment period provided definite evidence of a causal relationship between treatment and observed outcomes, including long-term protective effects against renal disease progression. Thus, intra-patient comparisons avoided inter-patient variability, which allowed adequately powered analyses despite the small sample size. The use of values of albuminuria averaged from measurements in three consecutive overnight urine collections minimized the confounding effect of random data fluctuations. The use of gold standard procedures for the direct measurement of GFR and RPF by inulin and $\mathrm{PAH}$ clearances, respectively, and the concomitant measurement of albumin and IgG fractional clearances were additional strengths. These data may be particularly relevant, since renal hemodynamics and sieving function has never been evaluated in the context of the Remission Clinic protocol. No previous study had evaluated the effect of this approach over such a long observation period.

\section{Acknowledgments}

This was a fully academic, internally funded study with no involvement of pharmaceutical companies. Dr. Antonietta Chianca performed the linear mixed-effects model. Manuela Passera helped prepare this article. P.C. is supported by a NIH T32 training grant (5T32AI078892-07).

\section{Disclosure Statement}

The authors have no conflicts of interest to disclose. 


\section{References}

1 Jais JP, Knebelmann B, Giatras I, De Marchi M, Rizzoni G, Renieri A, Weber M, Gross O, Netzer KO, Flinter F, et al: X-linked Alport syndrome: natural history in 195 families and genotype-phenotype correlations in males. J Am Soc Nephrol 2000;11:649-657.

-2 Gross O, Beirowski B, Koepke ML, Kuck J, Reiner M, Addicks K, Smyth N, Schulze-Lohoff E, Weber M: Preemptive ramipril therapy delays renal failure and reduces renal fibrosis in COL4A3-knockout mice with Alport syndrome. Kidney Int 2003;63:438-446.

$\checkmark 3$ Gross O, Licht C, Anders HJ, Hoppe B, Beck B, Tonshoff B, Hocker B, Wygoda S, Ehrich JH, Pape L, et al: Early angiotensin-converting enzyme inhibition in Alport syndrome delays renal failure and improves life expectancy. Kidney Int 2012;81:494-501.

-4 Remuzzi A, Malanchini B, Battaglia C, Bertani T, Remuzzi G: Comparison of the effects of angiotensin-converting enzyme inhibition and angiotensin II receptor blockade on the evolution of spontaneous glomerular injury in male MWF/Ztm rats. Exp Nephrol 1996;4: 19-25.

$\checkmark 5$ Barnett AH, Bain SC, Bouter P, Karlberg B, Madsbad S, Jervell J, Mustonen J: Angiotensinreceptor blockade versus converting-enzyme inhibition in type 2 diabetes and nephropathy. N Engl J Med 2004;351:1952-1961.

-6 Campbell R, Sangalli F, Perticucci E, Aros C, Viscarra C, Perna A, Remuzzi A, Bertocchi F, Fagiani L, Remuzzi G, Ruggenenti P: Effects of combined ACE inhibitor and angiotensin II antagonist treatment in human chronic nephropathies. Kidney Int 2003;63:1094-1103.

7 Rossing K, Christensen PK, Jensen BR, Parving $\mathrm{HH}$ : Dual blockade of the renin-angiotensin system in diabetic nephropathy: a randomized double-blind crossover study. Diabetes Care 2002;25:95-100.

8 Ruggenenti P, Perticucci E, Cravedi P, Gambara V, Costantini M, Sharma SK, Perna A, Remuzzi G: Role of remission clinics in the longitudinal treatment of CKD. J Am Soc Nephrol 2008;19:1213-1224.

$\checkmark 9$ Remission Clinic Task Force; Clinical Research Center 'Aldo e Cele Daccò'. The remission clinic approach to halt the progression of kidney disease. J Nephrol 2011;24:274-281.

10 Bakris GL, Weir M, De Quattro V, Rosendorff G, McMahon G: Renal hemodynamic and antiproteinuric response to an ACE inhibitor, trandolapril (T), or a calcium channel antagonist, verapamil $(\mathrm{V})$, alone or in fixed dose combination in patients with diabetic ne- phropathy: a randomized multicenter study. J Am Soc Nephrol 1996;7:1546.

1 Bianchi S, Bigazzi R, Caiazza A, Campese VM: A controlled, prospective study of the effects of atorvastatin on proteinuria and progression of kidney disease. Am J Kidney Dis 2003; 41:565-570.

12 Zoja C, Corna D, Camozzi D, Cattaneo D, Rottoli D, Batani C, Zanchi C, Abbate M, Remuzzi G: How to fully protect the kidney in a severe model of progressive nephropathy: a multidrug approach. J Am Soc Nephrol 2002; 13:2898-2908.

13 Ruggenenti P, Schieppati A, Remuzzi G: Progression, remission, regression of chronic renal diseases. Lancet 2001;357:1601-1608.

14 Anderson S: Systemic and glomerular hypertension in progressive renal disease. Kidney Int Suppl 1988;25:S119-S121.

15 Remuzzi A, Puntorieri S, Battaglia C, Bertani T, Remuzzi G: Angiotensin converting enzyme inhibition ameliorates glomerular filtration of macromolecules and water and lessens glomerular injury in the rat. J Clin Invest 1990;85:541-549.

16 Cravedi P, Ruggenenti P, Remuzzi G: Proteinuria should be used as a surrogate in CKD. Nat Rev Nephrol 2012;8:301-306. 